\title{
HIDDEN DOMAIN WALLS AS ELEMENTARY EXCITATIONS IN THE SPIN-1 VALENCE-BOND-SOLID MODEL
}

\author{
G. Fáth AND J. SóLyom
}

Research Institute for Solid State Physics, P.O. Box 49, 1525 Budapest, Hungary

Low-energy excitations are studied in the 1D $S=1$ antiferromagnetic valence-bond-solid model. The lowest states are found to form a discrete triplet branch, separated from a higher-lying scattering continuum. The elementary excitations are argued to be "hidden" domain walls.

PACS numbers: $75.10 . \mathrm{Jm}$

Antiferromagnetic spin-1 chains have been the subject of active research in the last ten years. The main motivation was to check Haldane's conjecture [1], who argued that the isotropic spin- $S$ Heisenberg antiferromagnets (HAF) should show drastically different ground-state properties depending whether $S$ is integer or half-integer. While these models with half-integer $S$ are generally massless, a massive phase (the Haldane phase) was proposed to emerge in the parameter space of couplings around the HAF point for all integer $S$, including the $S=1$ case. This phase is characterized by a unique ground state with exponential decay of the correlation functions and a finite energy gap to the excited states. Although we do not have a rigorous proof for Haldane's conjecture yet, there are lots of indirect evidences to support convincingly the predictions [2].

While the ground state in the Haldane phase is disordered in the conventional sense, there is some sort of hidden long-range order as it was revealed by den Nijs and Rommelse [3]. Introducing the nonlocal string order parameter $O_{\text {string }}^{\alpha}=$ $\lim _{|i-j| \rightarrow \infty}\left\langle\sigma_{i j}^{\alpha}\right\rangle, \alpha=x, y, z$, where $\langle\cdot\rangle$ denotes an expectation value in the ground state, and the string operator is defined by $\sigma_{i j}^{\alpha}:=-S_{i}^{\alpha} \exp \left[\mathrm{i} \pi \sum_{l=i+1}^{j-1} S_{l}^{\alpha}\right] S_{j}^{\alpha}$, they argued that $O_{\text {string }}^{\alpha}>0, \alpha=x, y, z$, in the Haldane phase. This prediction was later verified numerically by several authors.

The appearance of the hidden long-range order was further discussed by Kennedy and Tasaki [4], who showed that $O_{\text {string }}^{\alpha}>0$ corresponds to the spontaneous breaking of a hidden discrete $Z_{2} \times Z_{2}$ symmetry of the model. Using a nonlocal unitary transformation, they transformed the antiferromagnetic Hamiltonian into a ferromagnetic-like model with four ground states and explicit long-range order. As is generally expected, breaking of a discrete symmetry in the ground state leads to an excitation gap, since the Goldstone bosons do not appear. Excitations of the model can then be thought of as some kind of (hidden) domain 
walls, separating regions with the different ground states. Our main goal in this paper is to clarify this question.

The first rigorous example of a $S=1$ antiferromagnetic model in the Haldane phase is the valence-bond-solid (VBS or AKLT) model [5]:

$$
H=\sum_{j=1}^{N} h_{j, j+1}=\sum_{j=1}^{N}\left[\frac{1}{2} S_{j} \cdot S_{j+1}+\frac{1}{6}\left(S_{j} \cdot S_{j+1}\right)^{2}+\frac{1}{3}\right] .
$$

This also contains a biquadratic term besides the usual Heisenberg coupling. All the above-mentioned properties of the Haldane phase can be proven rigorously for this model [5]. Moreover, it is also possible to construct it exact ground state |VBS $\rangle$ from nearest-neighbor valence bonds.

We will use this model to study low-lying excitations in the Haldane phase. According to the conjecture the usual $S=1 \mathrm{HAF}$ is also expected to belong to this phase, therefore we think that the qualitative properties we will find for the VBS model should also characterize the Heisenberg model. Beside the fact that the ground state of the VBS model can be constructed analytically, there is another good reason to focus on this model. In a recent study of the general bilinear-biquadratic $S=1$ model, we observed that the convergence of various finite-size estimates to their thermodynamic limit is extremely fast in the close vicinity of the VBS point. This rapid convergence is not surprising, if we remember, that at this point first-neighbor valence bonds are only present, and the ground-state energy density becomes independent of the chain length. Although the excited states do show some dependence on the chain length $N$, this is found exponentially small for the most relevant levels. Therefore, finite-size calculations make possible to draw quite reliable conclusicns on the spectrum in the thermodynamic limit, which can then be compared directly to other analytical (variational) results.

Using a Lánczos algorithm we computed several low-lying eigenvalues of the VBS Hamiltonian with periodic boundary condition in each possible sector of momentum $k$ and also determined the total spin of the states. Chains with an even number of sites up to $N=16$ were considered. Figure 1 shows our results for the longest chain with $N=16$. Due to the fast convergence of the characteristic properties of the spectrum, the infinite chain limit can be inferred directly. (For more details see Ref. [6].) In the $|k| \geq \pi / 2$ range the lowest excitations form a continuous, one-parametric, discrete branch. All these states have total spin $S_{\mathrm{T}}=1$. Above this triplet branch the next higher excitations seem to build up a continuum. The triplet branch merges into this continuum somewhere under $k \sim \pi / 2$. Near $k=0$ there is no sign of the discrete curve, here the lowest-lying excitations are thought to belong to the continuum.

It is worth comparing these results to the known spectrum of integrable spin- $S$ chains, such as the spin-1/2 HAF. For those models it was shown [7] that the elementary excitations are in fact spin-1/2 solitons which are created always in singlet or triplet pairs due to topological reasons. The observable excitations thus form continua in $k$-space, and can be described by an even number of parameters. This is not the case for the spin-1 chain in the Haldane phase. The lack of such a multiparametric continuum for the lowest excited states shows that now the 


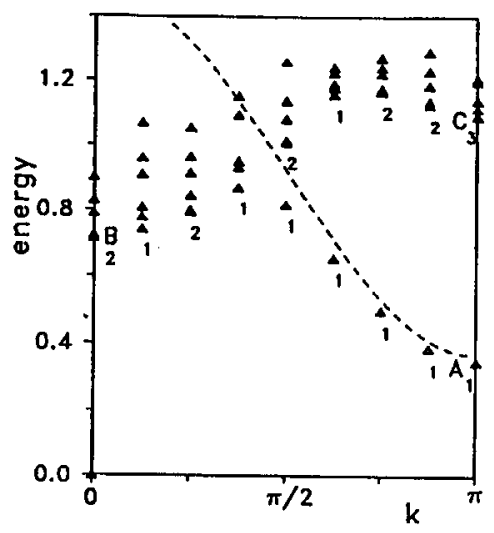

Fig. 1. Low-lying eigenvalues of the VBS model plotted vs. momentum $k$, for a chain with $N=16$ sites. Labels denote the total spin $S_{\mathrm{T}}$ of the states. Dashed line shows the energy of the trial state with a (hidden) domain wall.

elementary excitations are real spin-1, one-particle excitations. They cannot be decomposed into spin-1/2 particles.

There is another remarkable feature that can be observed in our finite chain calculation. The gap $\Delta_{B}$ to the lowest excited state of the $k=0$ subspace is approximately twice the singlet-triplet gap $\Delta_{A}$ at $k=\pi$. Similarly, the gap $\Delta_{C}$ between the ground state and the second excited state in the $k=\pi$ sector seems to be three times as large as the singlet-triplet gap. The physical picture behind such a behavior is simple. The excitations near $k=0$ can be composed of two low-lying excitations near $k=\pi$ and similarly, three excitations near $k=\pi$ can be combined to give another excitation near $k=\pi$. Analyzing more closely the lower boundary of the higher-lying continuum of excitations, it seems very likely that the two-particle states near $k=0$ are in fact the scattering states of two elementary excitations. The energy and momentum of such a state is simply the sum of the elementary energies and momenta, respectively, of the two particles. The situation is similar for the three-particle states near $k=\pi$. In the numerical calculation there does not seem to be any sign of bound states below the scattering continuum.

Now we will study the elementary excitations of the VBS model analytically. Our aim is to construct trial wave functions that contain "hidden" domain walls (solitons that destroy the hidden long-range order) and compare the calculated variational energy to the above numerical results. It will be seen that even in the simplest domain wall approach, the dispersion of the lowest triplet branch can be reproduced remarkably precisely.

The hidden order of the VBS ground state can be made explicit, if we use the nonlocal unitary transformation $U$ of Kennedy and Tasaki (see its definition in [4]). Under $U$, the two-site Hamiltonian $h_{j, j+1}, 1 \leq j \leq N-1$, transforms into a ferromagnetic-like two-site Hamiltonian $\tilde{h}_{j, j+1}$, whose ground-state sector with zero energy is four dimensional, and is spanned by the states $\phi_{\nu} \otimes \phi_{\nu}, \nu=1,2,3,4$, 
where the one-site states are defined as

$$
\begin{aligned}
& \phi_{1,2}=(|0\rangle \pm \sqrt{2}|+\rangle) / \sqrt{3}, \\
& \phi_{3,4}=(|0\rangle \pm \sqrt{2}|-\rangle) / \sqrt{3} .
\end{aligned}
$$

Note that this basis is not orthogonal, since $\left|\left\langle\phi_{\nu^{\prime}} \mid \phi_{\nu}\right\rangle\right|=1 / 3, \nu^{\prime} \neq \nu$. Unfortunately, because of the nonlocality of $U$, the Hamiltonian $h_{N, 1}$ does not transform into a similarly simple form. In order to avoid this subtlety, we turn to open boundary condition (BC) now. Note that we do not seek for exact solutions but variational results only, therefore the $\mathrm{BC}$ can have no relevance for long enough chains. However, it may cause some sort of inconveniences in the interpretation of our final results, as we will see soon.

Considering the transformed system $\widetilde{H}=\sum_{j=1}^{N-1} \tilde{h}_{j, j+1}$, its four ground states can be written as a simple tensor product

$$
\omega_{\nu}=\phi_{\nu} \otimes \phi_{\nu} \otimes \cdots \phi_{\nu} \otimes \phi_{\nu}, \quad \nu=1,2,3,4 .
$$

These states have long-range order reflecting the spontaneous breaking of a $Z_{2} \times Z_{2}$ symmetry, the only explicit (local) symmetry of $\widetilde{H}$. While $\left\langle S_{j}^{z}\right\rangle=+\frac{2}{3}$ for $\omega_{1}$ and $\omega_{2}$, we find $\left\langle S_{j}^{z}\right\rangle=-\frac{2}{3}$ for $\omega_{3}$ and $\omega_{4}$. Similarly, $\left\langle S_{j}^{x}\right\rangle=+\frac{2}{3}$ for $\omega_{1}$ and $\omega_{3}$, and $\left\langle S_{j}^{x}\right\rangle=-\frac{2}{3}$ for $\omega_{2}$ and $\omega_{4}$. The simplest domain wall is constructed by breaking the ground-state structure after a certain site $j$, and attached different ground states for the left and right parts as e.g. $|j\rangle=\phi_{1}^{1} \otimes \phi_{1}^{2} \otimes \ldots \phi_{1}^{j} \otimes \phi_{2}^{j+1} \otimes \phi_{2}^{j+2} \otimes \ldots \phi_{2}^{N}$.

To have a reasonable trial wave function, consider the linear combination

$$
|k\rangle=\sum_{j=1}^{N-1} \mathrm{e}^{\mathrm{i} k j}|j\rangle,
$$

where strictly speaking $k$ is rather a variational parameter than a momentum, because of the open boundary condition. In the thermodynamic limit, however, the BC should not count, and the variational energy that we obtain as a function of $k$ will in fact turn out to be the dispersion of excitations in this variational approach. (Note, there is still a possibility that the open BC brings a constant momentum shift $q$ in the final result, since we have the freedom to redefine $|j\rangle$ with e.g. an arbitrary phase factor $|j\rangle \rightarrow \mathrm{e}^{\mathrm{i} q j}|j\rangle$.)

To calculate the variational energy of the trial states $|k\rangle$, the basic quantities are $\left\langle j \mid j^{\prime}\right\rangle$ and $\left\langle j|\widetilde{H}| j^{\prime}\right\rangle$. However, in this case they are trivial because of the tensor product form of $|j\rangle$. Using the facts that $\left\langle\omega_{1} \mid \omega_{1}\right\rangle=\left\langle\omega_{2} \mid \omega_{2}\right\rangle=1$ and $\left\langle\omega_{1} \mid \omega_{2}\right\rangle=-1 / 3$, and the explicit form of $\tilde{H}$, one straightforwardly obtains $\left\langle j \mid j^{\prime}\right\rangle=(-1 / 3)^{\left|j-j^{\prime}\right|}$ and $\left\langle j|\tilde{H}| j^{\prime}\right\rangle=\delta_{j, j^{\prime}} 20 / 27$. Whence the variational energy yields

$$
\varepsilon(k)=\frac{\langle k|\widetilde{H}| k\rangle}{\langle k \mid k\rangle}=\frac{25+15 \cos (k)}{27} .
$$

This energy is plotted in Fig. 1 with a dashed line. As it is seen, the curve approximates the lowest triplet excitations quite convincingly, proving that the elementary excitations of the model can really be considered as moving (hidden) domain walls.

It is also very enlightening to transform back the trial states $|j\rangle$ into the original model $H$. It turns out [6] that they correspond to states, where the original 
VBS structure of the ground state is broken between sites $j$ and $j+1$, and the singlet bond (valence bond) is substituted by an appropriate triplet bond. This is nothing else but the "crackion" state of Knabe [8]. Although he did not analyse the momentum dependence of such trial states but gave only an upper bound for the primary gap of the model, his results can simply be generalized. After correcting an obvious mistake in the addition of numerical factors in his Eq. (3.10), our dispersion relation can readily be reproduced in his crackion approach.

Recently, another seemingly rather different trial wave function has been proposed to describe elementary excitations in the VBS model. Arovas, Auerbach and Haldane [9], using Feynman's single mode approximation analyzed the candidate excited states

$$
|k\rangle=N^{-1 / 2} \sum_{j=1}^{N} \mathrm{e}^{\mathrm{i} k j} S_{j}^{z}|\mathrm{VBS}\rangle
$$

and obtained exactly the same dispersion relation as that in Eq. (6). This is not surprising, however, because as we have shown it in Ref. [6], $S_{j}^{z}|\mathrm{VBS}\rangle$ is nothing but a linear combination of two crackion states with the triplet bonds between sites $j-1$ and $j$, and $j$ and $j+1$, respectively.

In summary, we have studied low-energy states of the spin-1 VBS model numerically and analytically in a variational approach. We have found that the elementary excitations are moving hidden domain walls, corresponding to triplet bonds in the VBS ground state. They form a discrete triplet branch under a scattering continuum in momentum space. Viewing the VBS model as a (small) perturbation of the spin-1 Heisenberg chain, we propose that excitations in the usual HAF should also have similar qualitative properties.

This research was supported in part by the Hungarian Research Fund (OTKA) grant Nos. T4473 and 2979. GF was also supported by the Hungarian Scientific Foundation.

\section{References}

[1] F.D.M. Haldane, Phys. Rev. Lett. 50, 1153 (1983); Phys. Lett. A 93, 464 (1983).

[2] For an overall review see I. Affleck, J. Phys. Condens. Matter 1, 3047 (1989).

[3] M. den Nijs, K. Rommelse, Phys. Rev. B 40, 4709 (1989).

[4] T. Kennedy, H. Tasaki, Phys. Rev. B 45, 304 (1992); Commun. Math. Phys. 147, 431 (1992).

[5] I. Affleck, T. Kennedy, E. Lieb, H. Tasaki, Phys. Rev. Lett. 59, 799 (1987); Commun. Math. Phys. 115, 477 (1988).

[6] G. Fáth, J. Sólyom, to be published.

[7] L.D. Faddeev, L.A. Takhtajan, Phys. Lett. A 85, 375 (1981); L.A. Takhtajan, Phys. Lett. A 87, 479 (1982).

[8] S. Knabe, J. Stat. Phys. 52, 627 (1988).

[9] D.P. Arovas, A. Auerbach, F.D.M. Haldane, Phys. Rev. Lett. 60, 531 (1988). 